\title{
Effects of lesions in hippocampus-entorhinal cortex on maze performance and activity in rats ${ }^{\prime}$
}

\author{
ROBERT M. BENDER, ${ }^{2}$ GAYLE HOSTETTER, ${ }^{3}$ AND \\ GARTH J. THOMAS, ${ }^{3}$ UNIVERSITY OF ILLINOIS
}

Five rats with lesions in the hippocampus plus entorhinal cortex, seven rats with lesions in the hippocampus that did not invade the entorhinal cortex, and 10 operated control rats were tested in an open field activity box and in a LashleyIII maze. Only Ss with lesions in hippocampus plus entorhinal cortex showed significantly enhanced activity, but both experimental groups were impaired in maze performance.

Research on behavioral effects of lesions in the hippocampus on activity level has led to inconsistent results. Lesion-induced increases in activity have been reported by a number of investigators using several different measures of activity. On the other hand, Hostetter \& Thomas (1967) did not find increased activity in an open field after hippocampal lesions, and Teitelbaum \& Milner (1963) reported that lesions in entorhinal cortex did not lead to enhanced activity. The inconsistent findings with regard to hippocampal lesions and activity suggest that differences in lesions that have escape reporting or differences in behavioral methods (or both) are of central significance.

Results from many studies have consistently shown that even very small lesions in the hippocampus markedly impair maze performance in several types of mazes (see Hostetter \& Thomas, 1967).

The purpose of the present study was to determine if caudally placed lesions that invaded the entorhinal cortex had any effects on open field activity level. Some of the lesions were mostly in the hippocampus and did not invade entorhinal cortex. The Ss were also tested for maze performance. Apparatus and Method

Twenty-two male albino rats (Holtzman) were used as Ss. The Ss were anesthetized with sodium pentobarbital. They were positioned in a Kopf stereotaxic instrument with bregma and lambda at the same vertical. In $12 \mathrm{Ss}$ experimental lesions were produced electrolytically by passing $2 \mathrm{~mA}$ anodal dc for 20 sec through the stereotaxically guided electrode aimed bilaterally at the cortex lying just caudal to the most posterior extent of the hippocampus. Ten operated control Ss underwent all stages of surgery except penetration of the brain by the electrode.

Activity level was determined in an open field box that has been previously described (Hostetter \& Thomas, 1967). On the 21 st postoperative day, each
$S$ was placed in the open field for $4 \mathrm{~min}$, and activity scores consisted of the total number of squares $\mathrm{S}$ entered by having all four feet within a square.

Maze learning was tested in a Lashley-III maze with guillotine doors at the entrance and exit of the maze and at all choice points to prevent retracing. After one week on a 23-1/2 $\mathrm{h}$ feeding schedule and 16 trials in a straight runway, the Ss were given five massed trials per day for at least six days (they were also trained to criterion). An error was counted each time $S$ passed an open door to the next alley by at least a body length. The performance score consisted of the total number of errors in 30 trials, excluding Trial 1.

After completion of behavioral testing, Ss were killed with an overdose of sodium pentobarbital and perfused percardially with physiological saline and $10 \%$ formalin. After further fixation in $10 \%$ formalin, the brains were embedded in celloidin, sectioned at $40 \mathrm{~m} \mu$, and every third section through the area of the lesion was stained with cresyl violet.

\section{Resulis}

Evaluation of the lesions in the histological material showed that we were not successful in confining the lesions to the most ventroposterior cerebral cortex (entorhinal; Krieg, 1946). In five brains the lesions involved both entorhinal cortex and posterior hippocampus. In seven brains the lesions were more anteriorly located and did not extend caudally into the entorhinal cortex. Behavioral data, therefore, are presented for these three groups: Group C, operated control, $N=10$, no lesions; Group $H-E$, experimental Ss with lesions in the hippocampus that also invaded the entorhinal cortex, $\mathrm{N}=5$; and Group $\mathrm{H}$, experimental Ss with lesions in the hippocampus that did not invade the entorhinal cortex, $N=7$.

Activity scores are shown in Table 1 (medians and ranges). Kruskal-Wallis $H$ test (one-way analysis of variance by ranks) indicated significant differences among groups $(H=72.1, p<.001)$. $U$ tests showed

Table 1. Activity (squares entered)

\begin{tabular}{lccc} 
& Control Group $(\mathrm{N}=10)$ & Group H-E $(\mathrm{N}=5)$ & Group $\mathrm{H}(\mathrm{N}=7)$ \\
\hline Medians & 92 & 166 & 121 \\
Ranges & $52-131$ & $65-191$ & $36-202$ \\
& Maze (errors in trials 2 through 30) & \\
Median s & 40 & 105 & 67 \\
Ranges & $22-67$ & $67-157$ & $39-86$ \\
\hline
\end{tabular}




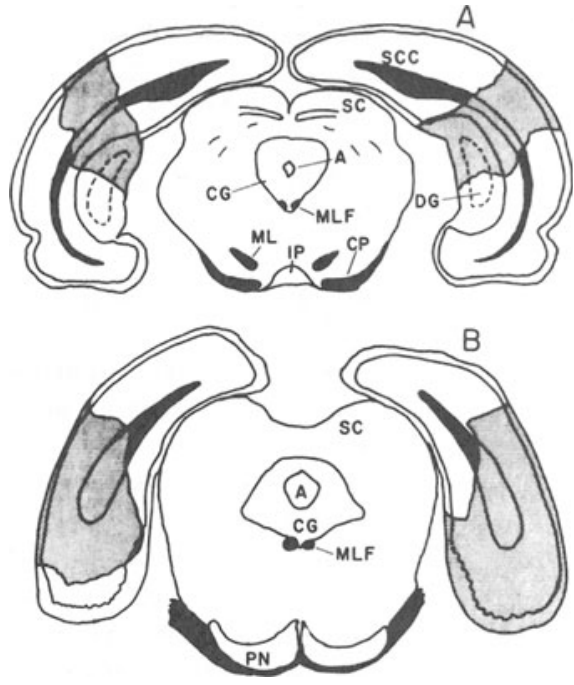

Fig. 1. Tracings to illustrate lesions. Panel $A$ shows lesions from Group H, and Panel B shows lesions from Group H-E. Abbreviations: A, aqueduct of Sylvius; CG, central gray; CP, cerebral peduncle; DG, dentate gyrus of the hippocampal formation; IP, interpeduncular nucleus; ML, medial lemniscus; MLF, medial longitudinal fasciculus; PN, pontine nuclei; SCC, splenium of the corpus callosum; and SC, superior colliculus.

that Group H-E entered more squares than did Group C ( $<<.05)$, but Group $H$ did not differ significantly from control Ss (Group C) or from the other experimental group (Group H-E). The median score of Group $H$ is much greater than the median of Group $C$, but not significantly so. The striking effect of the lesions in Group $H$ was the large variability within the group indicated by the range of activity scores in Table $1(36-202)$. The effect of the lesions on activity in Group $\mathrm{H}-\mathrm{E}$ was more uniform, and the group was significantly more active than the control group.

As has been shown many times (see Hostetter \& Thomas, 1967), damage to the hippocampus impairs maze performance. Nonparametric one-way analysis of varlance (Kruskal-Wallis $H$ test) of the three groups of error scores shown in Table 1 is highly significant $(H=91.6, \quad p<.001)$. $U$ tests showed that each experimental group differed significantly from the control group (for Group $\mathrm{H}-\mathrm{E}, \mathrm{p}<.001$; for Group H, $p<.01)$. The two experimental groups did not differ significantly from each other.

Evaluation of the lesions showed that they were very similar in overall size. They differed in location mostly in the rostrocaudal direction. Lesions in Group $\mathrm{H}-\mathrm{E}$ were simply more caudally situated than lesions in Group H. Damage to the hippocampus was limited to the posterolateral portion, but there was damage to the dentate gyrus as well as to neo- cortex, corpus callosum, and medullary layer (along the electrode tracks). Figure 1 shows tracings from selected sections to illustrate the lesions: Panel A shows lesions that did not invade the entorhinal cortex (from Group H), and Panel B shows more caudally located lesions that involved both posterior hippocampus and entorhinal cortex (from Group H-E). No consistent relationship could be found between various individual features of the lesions and behavioral scores on either the activity test or the maze-learning test. Rank-order correlation between overall size of the lesions, regardless of location, and indices of performance was not significant.

Discussion

The results suggest that "hippocampal" lesions must extend into posterior cortex (entorhinal) in order to cause the marked effect on open field activity level that has been reported to follow hippocampal lesions. This conclusion seems to be in direct contradiction to the report of Teitelbaum \& Milner (1963) who found decreased activity after ablation of entorhinal cortex. There are, however, great differences (and probably significant ones) in procedure between their experiment and the present one. Their activity box was much smaller than ours, and their Ss were under food deprivation. It seems likely that "activity level" comprises very complicated processes neurologically and behaviorally, and effects of lesions might be task specific as well as interacting significantly with the intrinsic activity cycles of rats.

The finding that lesions of the hippocampus markedly impair maze performance is in agreement with results of many previous studies. The present study does not further our understanding of this lesioninduced behavioral dysfunction.

\section{References}

HOSTETTER, GAYLE, \& THOMAS, G. J. Evaluation of enhanced thigmotaxis as a condition of impaired maze learning by rats with hippocampal lesions. J. comp. physiol Psychol, 1967, 63, 105-110.

KRIEG, W. J. S. Connections of the cerebral cortex. I. The albino rat. A. Topography of the cortical areas. J. comp. Neurol, 1946, 84, 221-275.

TEITELBAUM, H., \& MILNER, P. Activity changes following partial hippocampal lesions in rats. J. comp. physiol Psychol, 1963, 56, 284-289.

\section{Notes}

1. This study is based on part of an honors thesis by the senior author, supported in part by the National Science Foundation Research Participation Program at the University of Illinois and in part by Grant 1370 from the National Institute of Mental Health. The authors are indebted to Ruth V. Chalmers for preparing the histological materials. 2. Present address: Marquette University School of Medicine, Milwaukee, Wisconsin, 53233.

3. Present address: Center for Brain Research, University of Rochester, Rochester, New York, 14627. 